\title{
Quantum Mechanical Calculation of Molecular Radii. I. Hydrides of Elements of Periodic Groups IV through VII
}

\author{
CARL W. KaMmEYer \\ Department of Chemistry, The University of Michigan-Flint, Flint, Michigan 48503 \\ AND \\ DONAID R. WHITMAN \\ Department of Chemistry, Case Western Reserve University, Cleveland, Ohio 44106
}

(Received 26 November 1971)

\begin{abstract}
The fraction of the total electron density within a sphere having the empirical van der Waals radius is calculated for the atoms of eight elements, using Hartree-Fock atomic wavefunctions. The sphere is found to contain $97 \%-99 \%$ of the electron density, indicating that such calculations correlate well with atomic sizes. The procedure is extended to eight hydride molecules of the type $\mathrm{AH}_{n}$, with molecular size determined by the radius of the $98 \%$ contour as computed from published one-center SCF molecular wavefunctions. The predicted molecular radii agree well with experimental values and support the usefulness of this method for describing effective molecular size.
\end{abstract}

\section{INTRODUCTION}

Traditionally, the sizes of atoms have often been described by their covalent or ionic radii. However, these sizes can also be expressed in terms of van der Waals radii, determined empirically from the equilibrium internuclear distances between atoms which are in contact but not chemically bonded. It is these van der Waals radii, as given in Pauling's widely reproduced table, ${ }^{1}$ that are used for the dimensions of commercial models. Experimental van der Waals radii can also be found for molecules, assuming effective sphericity, from such properties as gas viscosities and interplanar spacings in crystals.

The determination of atomic and molecular sizes from electron-density calculations has become feasible, now that high-quality wavefunctions are available for atoms and, increasingly, for molecules. Bader, Cade et al..$^{2}$ have obtained dimensions for a number of diatomic molecules, choosing, as a measure of molecular size, the contour with density 0.002 electrons per cubic bohr. An even more simple and intuitive approach to molecular size is to define an "effective molecular radius" as the radius of that spherical surface which encloses a certain fraction of the total electron density. Correlation, for atoms, of Hartree-Fock electron density with empirical van der Waals radius indicates that the appropriate value of this fraction is $98 \%$.

\section{ATOMS}

Calculations of electron densities for atoms were performed with Clementi's Roothaan-Hartree-Fock wavefunctions. ${ }^{3}$ In these wavefunctions each atomic orbital is a linear combination of normalized Slater-type orbitals with optimized exponents.

For each atomic orbital, the spherically averaged electron density was integrated from the origin to a distance $R$, to obtain the fraction of the orbital density within the sphere of radius $R$. The mean of these orbital density fractions, each weighted according to orbital occupancy in the atom, is the fraction of the total electron density within the sphere. This "spherical density fraction," $f(R)$, was specifically evaluated at the empirical van der Waals radius for each of eight nonmetallic elements. The radii are those given by Rich, ${ }^{4}$ which are slightly modified from the earlier Pauling values. The results, shown in Fig. 1, indicate that for neutral atoms the van der Waals sphere contains between $97 \%$ and $99 \%$ of the Hartree-Fock electron density.

The electron density fraction within the van der Waals sphere is found to increase from left to right

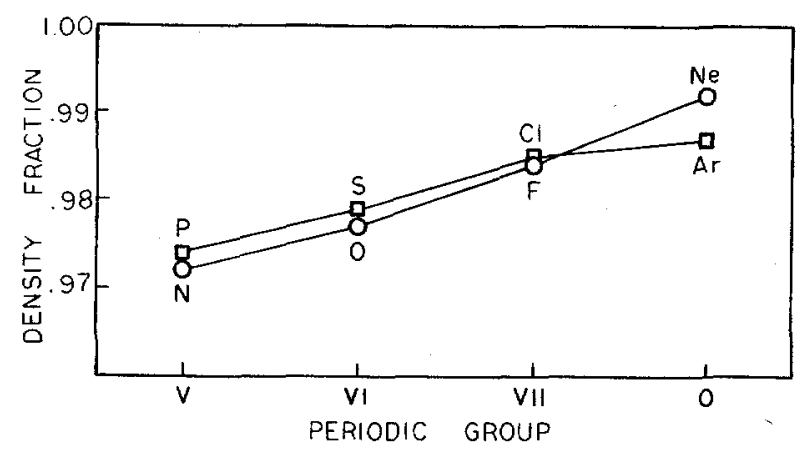

Fig. 1. Fractions of the Hartree-Fock electron density within the sphere of van der Waals radius (Ref. 4) for neutral atoms.

within a period. This increase in "compactness" is fully consistent with the concept of filling an electron shell. The larger density fractions for atoms of second-period elements (with the exception of the anomalous results for neon and argon) are consistent with the concept of inner shell electrons, which are of course more numerous in elements of higher atomic number.

The empirical van der Waals atomic radii are similar to, and in most cases estimated from, ionic radii, "inasmuch as the bonded atom presents the same face to the outside world in directions away from its bond as the ion does in all directions." However, a possible 


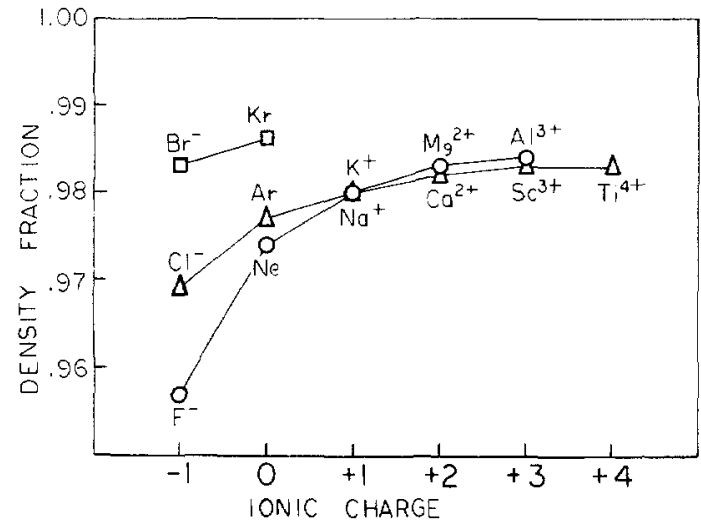

FIG. 2. Fractions of the Hartree-Fock electron density within the sphere of radius equal to the "univalent radius" (Ref. 6) of the ion.

alternative to van der Waals radii, which might remove some of the intraperiod variation, is provided by Pauling's "univalent radii," 6 wich represent the sizes multivalent ions would exhibit if their Coulomb interactions were univalent. Results for Hartree-Fock electron-density fractions within "univalent radius" spheres are shown in Fig. 2. There is again some variation, yet all the values are near $98 \%$. In this case, moreover, the expected ordering of neon and argon is observed.

The sensitivity of the density fraction to the quality of the atomic basis set was tested by repeating the calculations, but using Clementi's double-zeta wavefunctions, ${ }^{3}$ which have much smaller basis sets than do the Hartree-Fock wavefunctions. It is reassuring that excellent agreement is obtained from different size basis sets as shown in Table I.

It can validly be concluded that the radius of a sphere containing $98 \%$ of the total electron density correlates well with the effective size of an atom.

\section{MOLECULES}

The van der Waals radius concept can be extended to include many diatomic and polyatomic molecules by

TABLE I. Fractions of total electron density within sphere of van der Waals radius, for wavefunctions of different quality.

\begin{tabular}{llcc}
\hline & \multicolumn{2}{c}{ Fraction } \\
\cline { 3 - 4 } Element & $\begin{array}{c}\text { Wadius } \\
\text { (angstrom) }\end{array}$ & $\begin{array}{c}\text { Wavefunction } \\
\text { with ab- } \\
\text { breviated } \\
\text { basis set }\end{array}$ & $\begin{array}{c}\text { Hartree-Fock } \\
\text { wavefunction }\end{array}$ \\
\hline Nitrogen & 1.5 & 0.974 & 0.972 \\
Oxygen & 1.40 & 0.979 & 0.977 \\
Fluorine & 1.35 & 0.986 & 0.983 \\
Phosphorus & 1.9 & 0.975 & 0.974 \\
Sulfur & 1.85 & 0.980 & 0.980 \\
\hline \hline
\end{tabular}

\footnotetext{
a Reference 4
}

${ }^{b}$ Reference 3 .

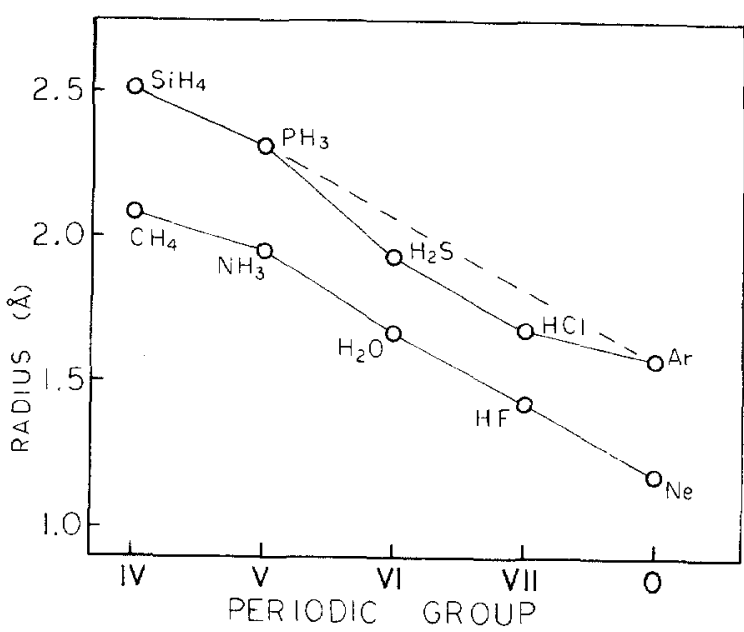

Fig. 3. Effective molecular radii of hydrides (Moccia's wavefunctions). Also radii of the isoelectronic inert gas atoms calculated by the same precedure.

assuming that they can be described as effectively spherical. The "effective molecular radius," then, is defined as that of the sphere containing $98 \%$ of the total electron density.

This approach is particularly applicable to hydride molecules of the type $\mathrm{AH}_{n}$, which have nearly spherical electron distributions. Such molecules are described conveniently by one-center-expansion (OCE) wavefunctions, in which all the basis orbitals are centered at the heavy nucleus. Moccia ${ }^{7}$ has determined selfconsistent-field OCE wavefunctions for the hydrides $\mathrm{CH}_{4}$ through $\mathrm{HF}$, and $\mathrm{SiH}_{4}$ through $\mathrm{HCl}$, using basis sets of up to 30 Slater-type orbitals with $l \leq 3$.

Effective molecular radii were calculated for these eight molecules, using Moccia's wavefunctions, with results as shown in Fig. 3 along with atomic radii of the isoelectronic inert gas atoms calculated by the same procedure. The radii for the 10 -electron molecules vary smoothly from $2.08 \AA$ for methane to $1.43 \AA$ for hydrogen fluoride and $1.18 \AA$ for neon. The values for the 18-electron molecules are less consistent, probably due to the relatively poorer quality of Moccia's wavefunctions for these larger molecules. For $\mathrm{H}_{2} \mathrm{~S}$ and $\mathrm{HCl}$, Moccia's wavefunctions are expanded in basis sets of the same size as for the corresponding 10-electron mole-

TABLE II. Effective radii of the ammonia molecule calculated for different one-center-expansion wavefunctions.

\begin{tabular}{cc}
\hline \hline Basis set & Radius $(\AA)$ \\
\hline Moccia, $^{\text {a }} 7 \times 4 \times 4$ & 1.86 \\
Moccia $^{\mathrm{b}} 12 \times 9 \times 9$ & 1.95 \\
Joshi, $^{\mathrm{c}} 13 \times 12 \times 12$ & 1.89 \\
\hline
\end{tabular}

Truncated from $b$.

b Reference 7 .

c B. Joshi, J. Chem. Phys. Suppl. 43, S40 (1965), Table VI. 
TABLE III. Comparison of calculated effective radii with experimental crystal radii and with theoretical dimensions of corresponding monohydrides (all distances in angstroms).

\begin{tabular}{|c|c|c|c|c|c|c|}
\hline \multicolumn{3}{|c|}{ Polyhydrides } & \multirow{2}{*}{\multicolumn{4}{|c|}{ Monohydrides }} \\
\hline \multirow[b]{2}{*}{ Molecule } & \multirow{2}{*}{$\begin{array}{l}\text { Calculated } \\
\text { radius }\end{array}$} & \multirow{2}{*}{$\begin{array}{l}\text { Crystal } \\
\text { radius }^{\mathrm{a}}\end{array}$} & & & & \\
\hline & & & Molecule & $L / 2$ & $r_{A}$ & Ref. \\
\hline $\mathrm{CH}_{4}$ & 2.08 & 2.06 & $\mathrm{CH}$ & 2.10 & 1.85 & $2(b)$ \\
\hline $\mathrm{NH}_{3}$ & 1.95 & 1.82 & $\mathrm{NH}$ & 1.92 & 1.69 & $2(b)$ \\
\hline $\mathrm{H}_{2} \mathrm{O}$ & 1.67 & $1.38^{\mathrm{b}}$ & $\mathrm{OH}$ & 1.78 & 1.53 & $2(\mathrm{~b})$ \\
\hline $\mathrm{HF}$ & 1.43 & $1.25^{\mathrm{b}}$ & HF & 1.68 & 1.43 & $2(\mathrm{~b})$ \\
\hline $\mathrm{SiH}_{4}$ & 2.51 & & $\mathrm{SiH}$ & 2.49 & 2.17 & $2(d)$ \\
\hline $\mathrm{PH}_{3}$ & 2.31 & 2.23 & $\mathrm{PH}$ & 2.33 & 2.01 & $2(d)$ \\
\hline $\mathrm{H}_{2} \mathrm{~S}$ & 1.93 & 2.04 & SH & 2.17 & 1.91 & $2(d)$ \\
\hline $\mathrm{HCl}$ & 1.68 & & $\mathrm{HCl}$ & 2.04 & 1.75 & $2(d)$ \\
\hline
\end{tabular}

a See Footnote 9.

cules, with a consequent loss in quality for the outer molecular orbitals, due to their higher energy.

A direct illustration of the influence of the basis set upon the calculated molecular size is shown in Table II. The effective radius of the ammonia molecule was calculated, using the $98 \%$ criterion, for three SCF OCE wavefunctions with different basis sets. Although the variation in results is not great, it indicates that care is necessary in selecting basis orbitals to ensure a good description of the electron distribution in the surface portion of the molecule. This is due to the insensitivity of the total energy to details of the electron distribution in regions distant from the nuclei.

The results found here agree well with published values determined by various methods. Pauling's ${ }^{8}$ estimate of $2.0 \AA$ for the radius of the methyl and the methylene groups provides an empirical value with which the calculated radius for methane $(2.08 \AA)$ compares favorably.

The predicted molecular radii correlate closely with experimental values obtained from crystal lattice spacings, ${ }^{9}$ as shown in Table III. The effective radii are slightly larger than the crystal radii, except for hydrogen sulfide, where again the smaller calculated size is attributable to the relatively poorer quality of the wavefunction. The differences are about $0.1 \AA$, except for water and hydrogen fluoride, where hydrogen bonds produce comparatively smaller crystal radii. The generally good agreement clearly demonstrates the validity of the calculated values.

Bader, Cade et al..$^{2 b, 2 d}$ describe the calculated size of a pear-shaped monohydride molecule $\mathrm{AH}$ in terms of its length $L$ and the radius $r_{A}$ of atom $\mathrm{A}$ in the molecule. The molecular radii obtained here for polyhydrides can be compared with their values of $L / 2$ and $r_{\mathbf{A}}$, also shown in Table III. For both the 10 - and the 18 electron molecules, the effective radius for the tetrahydride is near their value of $L / 2$ for the corresponding monohydride. The effective radii decrease regularly to
${ }^{b}$ There are hydrogen bonds in these crystals.

a value for the monohydride which is near their $r_{A}$. This trend is in accord with the decrease in sphericity of the polyhydride molecules.

\section{CONCLUSIONS}

The method employed for calculating effective molecular sizes invokes the assumption of a sphere enclosing a constant fraction of the total electron density. This assumption of sphericity is in keeping with the recognized principle that the usefulness of a model decreases rapidly as the number of its empirical parameters increases. The method is conceptually reasonable, computationally simple, and yields successful predictions. In principle, it can be applied to any molecule for which a molecular orbital wavefunction is available, although additional conceptual and computational complexities arise with multicenter wavefunctions. It is appropriate to conclude that effective sizes of molecules can be correlated with electron densities as calculated from SCF MO wavefunctions.

${ }^{1}$ L. Pauling, The Nature of the Chemical Bond (Cornell U. P., Ithaca, N.Y., 1960), 3rd ed., p. 260.

2. (a) R. F. W. Bader, W. H. Henneker, and P. E. Cade, J. Chem. Phys. 46, 3341 (1967); (b) R. F. W. Bader, I. Keaveny and P. E. Cade, ibid. 47, 3381 (1967); (c) R. F. W. Bader and A. D. Bandrauk, ibid, 49,1653 (1968); (d) P. E. Cade, R. F. W Bader, W. H. Henneker, and I. Keaveny, ibid. 50, 5313 (1969); and (e) P. E. Cade, R. F. W. Bader, and J. Pelletier, ibid. 54, 3517 (1971).

${ }^{3}$ E. Clementi, IBM J. Res. Develop. 9, 2 (1965).

${ }^{4}$ R. Rich, Periodic Correlations (Benjamin, New York, 1965), p. 36.

${ }^{5}$ L. Pauling, Ref. 1, p. 258.

${ }^{6}$ Reference 1, pp. 513-514.

${ }^{7}$ R. Moccia, J. Chem. Phys. 40, 2164, 2176, 2186 (1964).

${ }^{8}$ L. Pauling, Ref. 1, p. 261.

${ }^{2}$ For $\mathrm{CH}_{4}, \mathrm{NH}_{3}, \mathrm{PH}_{3}$, and $\mathrm{H}_{2} \mathrm{~S}$, the crystal radii are calculated from the length of the edge of the unit cell, assumed to be facecentered cubic. For $\mathrm{H}_{2} \mathrm{O}$ and $\mathrm{HF}$, the radii are taken to be onehalf of the $\mathrm{O}-\mathrm{O}$ and $\mathrm{F}-\mathrm{F}$ distances. Values for $\mathrm{SiH}_{4}$ and $\mathrm{HCl}$ were not available. Data, except for $\mathrm{H}_{2} \mathrm{O}$, were taken from $\mathrm{R}$. W. G. Wyckoff, Crystal Structures (Interscience, New York, 1966), 2 nd ed., Vols. 1-5. The $\mathrm{O}-\mathrm{O}$ distance in ice is from Pauling, Ref. 1, p. 465 . 